\title{
General Characteristics of Free-Return Ensured Orbit Insertion and Trajectory Design with MOI Robustness in MMX Mission
}

\author{
By Shota TAKAHASHI, ${ }^{1)}$ Naoko OGaWA, ${ }^{2)}$ and Yasuhiro KaWAKATSU ${ }^{2)}$ \\ 1) Department of Space and Astronautical Science, SOKENDAI (The Graduate University for Advanced Studies), Hayama, Japan \\ ${ }^{2)}$ Institute of Space and Astronautical Science, JAXA, Sagamihara, Japan
}

(Received June 30th, 2017)

\begin{abstract}
Failure of the orbit insertion maneuver has a significant impact on the entire mission, for the trajectory of a spacecraft is largely deflected by swing-by. The risk can be reduced by targeting a point on the B-plane where the spacecraft reaches the free-return (FR) trajectory with the target body in the case of insertion failure. The backup orbit must also satisfy conditions suitable for the mission. We investigated the type of orbit insertion that is both robust to failure and reasonable for the mission requirements. We call this method FR ensured orbit insertion. Among various failure modes of the orbit insertion maneuver, we focus on the complete maneuver failure. The impact parameters on the B-plane to achieve the orbit insertion are formulated based on the geometry of velocity vectors at swing-by. The necessary deflection angle $\alpha_{F R}$ at swing-by must be smaller than the possible maximum deflection angle $\alpha_{\max }$ for the target body. When we introduce proper scaling factors, the relation of $\alpha_{\max }$ and $\alpha_{F R}$ is characterized by a single parameter $\lambda$. Using polar orbit insertion as an example, maps which show the reachability of FR trajectory after the insertion failure for each approaching condition are presented. The derived maps can be used as a tool to assess the applicability of the method in the mission design. Finally, as an application to practical mission design, we demonstrate the use of FR ensured orbit insertion in JAXA's MMX mission.
\end{abstract}

Key Words: Free-Return, Orbit Insertion, Swing-by, MMX

\section{Nomenclature}

a : semi-major axis

$b \quad: \quad$ norm of $\boldsymbol{B}$ vector

$D(\alpha)_{n} \quad$ : $\quad$ rotation matrix around $\mathrm{n}$-th axis by angle $\alpha$

$\hat{\boldsymbol{k}} \quad: \quad$ reference vector to represent orbital plane

$L_{s f} \quad$ : scaling factor for length

$M \quad$ : number of revolution by a target body

$N \quad$ : number of revolution by a spacecraft

$\hat{\boldsymbol{S}}, \hat{\boldsymbol{T}}, \hat{\boldsymbol{R}} \quad$ : $\quad$ unit vectors for B-plane coordinate system

$T \quad$ : orbital period

$T_{s f} \quad: \quad$ scaling factor for time

$v_{\infty} \quad:$ hyperbolic excess velocity

$\alpha \quad: \quad$ deflection angle at swing-by

$\beta \quad: \quad$ angle between $\boldsymbol{v}_{\text {oin }}$ and $-\boldsymbol{v}_{\boldsymbol{p}}$

$\delta \quad:$ declination

$\eta \quad: \quad$ angle between $\boldsymbol{v}_{\boldsymbol{p}}$ and $\boldsymbol{v}_{\text {out }}$

$\theta \quad$ : angle from $\hat{\boldsymbol{T}}$ axis to $\boldsymbol{B}$

$\lambda \quad:$ non-dimensional parameter

$\mu \quad: \quad$ standard gravitational parameter

$\varphi_{R} \quad: \quad$ angle from $\hat{\boldsymbol{P}}_{\mathbf{1}}$ axis to $\hat{\boldsymbol{R}}$ axis

$\psi \quad$ : parameter to express $\boldsymbol{v}_{\text {cout }}$

$\omega \quad$ : argument of periapsis

Subscripts

in : incoming

out : outgoing

$p \quad:$ planet

Superscripts

*

* non-dimensional

unit vector

\section{Introduction}

In interplanetary exploration, the orbit insertion is one of the most critical events in the entire mission. If an orbiter fails in the maneuver to decelerate itself relative to a target body, it leads to unintended swing-by. Therefore, the failure of the orbit insertion has a substantial impact on the outcome of a mission.

Generally, there are a lot of failure modes in the orbit insertion maneuver. In NASA's NEAR Shoemaker mission, a temporary malfunction in the attitude control system during a maneuver caused one-year delay of the rendezvous with the

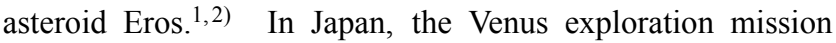
Akatsuki suffered from the insertion failure. The orbit maneuvering engine broke down amid the thrust and the achieved $\Delta V$ was only $20 \%$ of its nominal value. ${ }^{3)}$ In order to assess the possibility of each failure mode, consideration of thrusters and spacecraft systems would be necessary. In this study, we only focus on the failure mode where the spacecraft cannot perform any maneuver and is lost in deep space.

As a measure to avoid the risk, utilization of the free-return (FR) trajectory has been proposed. ${ }^{4}$ The idea is to target a point on the B-plane so that a spacecraft enters an FR trajectory with the target body when the maneuver for insertion fails. If a spacecraft is on the FR trajectory, the spacecraft's and target body's revolution period around the primary body make a simple integer ratio, which materializes the re-encounter. The previous work has focused only on the trajectory design for the SELENE mission by JAXA. Thus, the motivation of this study is to investigate the applicability of the method in general. As a first step, we formulate the B-plane parameters $b$ and $\theta$ necessary for a spacecraft to reach FR trajectories, which has not been shown in the previous works. The formulation is based on the geometric analysis of the velocity vectors at swing-by. 


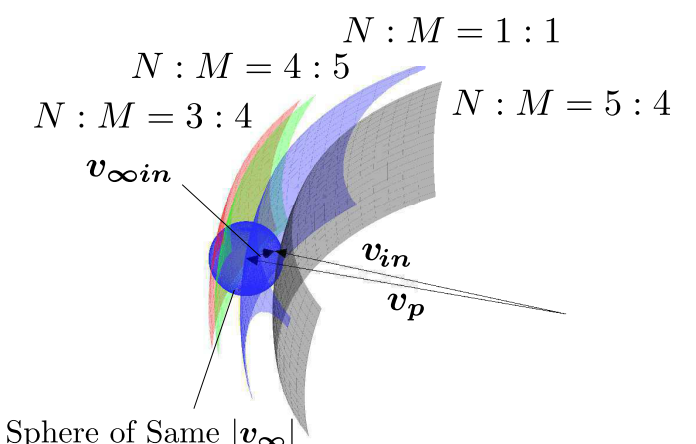

Sphere of Same $\left|\boldsymbol{v}_{\infty}\right|$

Fig. 1. 3D view of the velocity vector geometry at swing-by.

When we use an FR trajectory as a backup trajectory, we must ensure that the orbit after the successful insertion is suitable in terms of mission requirements. For instance, we require a spacecraft to have small insertion $\Delta V$ or pass through a particular point over the target body. Therefore, our concern is whether we can prepare the backup trajectory without much changing of the original conditions for insertion. Another contribution of this study is to systematically investigate the approaching conditions that will lead to FR trajectories satisfying mission requirements. Using polar orbit insertion as an example, maps are presented which show the reachability and information regarding insertion $\Delta V$ over the grid of approaching condition.

To further evaluate if the method can be utilized in a practical mission design with a complex insertion sequence, the application to JAXA's MMX (Martian Moons eXploration) mission is studied. This is a sample return mission planned to be launched in the early 2020s. The spacecraft needs to perform multiple maneuvers for Mars orbit insertion (MOI), which inserts the probe into the same orbit with one of the Martian moons Phobos. When we draw a contour of the distribution of $\Delta V$ for MOI on the B-plane, it shows a complex trend. The process to design a trajectory is discussed that satisfies mission requirements and the FR conditions.

\section{Geometric Analysis of Velocity Vectors}

\subsection{Velocity to achieve free-return trajectory}

We assume a target body and a primary body as a planet and the Sun respectively for ease of explanation. The insertion to the FR trajectory after the swing-by is realized in other systems such as the Earth-Moon system in the same manner. As for the swing-by trajectory where a spacecraft naturally returns to the same body after a certain time of flight, the exhaustive research has been conducted in the framework of a two-body problem. ${ }^{5}$ ) Of the possible FR trajectories presented in the research, we focus only on the full-revolution trajectories. The FR trajectory with the target body satisfies the following relation.

$$
T_{S / C}=\frac{M}{N} T_{p}
$$

where $N$ and $M$ are irreducible. A spacecraft can re-encounter the target body at the same location after the period of $M T_{p}$ in the case of insertion failure. Figure 1 shows the relation among velocity vectors at the swing-by. Since $\left|\boldsymbol{v}_{\infty \text { in }}\right|$ equals $\left|\boldsymbol{v}_{\infty \text { out }}\right|$, the vector $\boldsymbol{v}_{\infty \text { out }}$ is somewhere on the sphere with radius $\left|\boldsymbol{v}_{\infty}\right|$. When $\boldsymbol{v}_{\infty \text { out }}$ is at the intersection of $\left|\boldsymbol{v}_{\infty}\right|$ sphere and the sphere whose radius is expressed in Eq. (2), the necessary condition for FR ensured orbit insertion is met.

$$
v_{\text {out }}=\sqrt{\mu_{\text {Sun }}\left(\frac{2}{r_{p}}-\left(\frac{N}{M}\right)^{\frac{2}{3}} \frac{1}{a_{p}}\right)}
$$

\subsection{B-plane parameters for $\mathbf{F R}$ trajectories}

Several formulas and coordinate systems are proposed to help design swing-by trajectories. ${ }^{6,7)}$ They are useful when we consider multiple variables at the same time such as orbital period, inclination, eccentricity, etc. However, our objective is to find the relation between B-plane parameters and orbital period; therefore, new reference frames are introduced. Figures 2 and 3 show the definition of angles and coordinate systems associated with the velocity vectors. The point $O_{1}$ is the origin of the velocity vectors viewed from the primary body, whereas the point $\mathrm{O}_{2}$ is the origin of the velocity vectors viewed from the planet. The orthogonal coordinate system $O_{2}-q_{1} q_{2} q_{3}$ and $O_{2}-P_{1} P_{2} P_{3}$ are defined. The direction of $q_{1}$ axis is same with $\boldsymbol{v}_{\boldsymbol{p}}$. The direction of $q_{2}$ and $P_{1}$ is the same as the $\boldsymbol{v}_{\boldsymbol{i n}} \times \boldsymbol{v}_{\boldsymbol{p}}$ direction. $P_{2}$ is aligned with $\boldsymbol{v}_{\infty \boldsymbol{i n}}$. The vector $\boldsymbol{v}_{\boldsymbol{c} \text { out }}$ comes at the intersection of $v_{\infty}$ sphere and the sphere of $\left|\boldsymbol{v}_{\text {out }}\right|$. The intersection of the two spheres becomes a circle, and we can designate a $\boldsymbol{v}_{\text {oout }}$ by a single parameter $\psi$ as in Fig. 4 .

$$
\boldsymbol{v}_{\text {cout }}=\left(\begin{array}{c}
L \\
V \sin \psi \\
V \cos \psi
\end{array}\right)
$$

$L$ and $V$ are expressed as follows.

$$
\begin{aligned}
L & =v_{\text {out }} \cos \eta-v_{p}, \\
V & =v_{\text {out }} \sin \eta, \\
\text { where } \quad \eta & =\arccos \left(\frac{v_{p}^{2}+v_{\text {out }}^{2}-v_{\infty}^{2}}{2 v_{p} v_{\text {out }}}\right) .
\end{aligned}
$$

By rotating the coordinate system, the expression for $\boldsymbol{v}_{\infty \text { out }}$ in the $O_{2}-P_{1} P_{2} P_{3}$ coordinate system is derived.

$$
\begin{aligned}
\boldsymbol{v}_{\text {⿻out }} & =D(\beta)_{1} D(\pi / 2)_{3}\left(\begin{array}{c}
L \\
V \sin \psi \\
V \cos \psi
\end{array}\right) \\
& =\left(\begin{array}{c}
V \sin \psi \\
V \sin \beta \cos \psi-L \cos \beta \\
L \sin \beta+V \cos \beta \cos \psi
\end{array}\right)
\end{aligned}
$$

From this equation, a deflection angle $\alpha$ after the swing-by for each parameter $\psi$ is obtained.

$$
\begin{aligned}
2 \alpha & =\arccos \left(\frac{\boldsymbol{v}_{\text {कin }} \cdot \boldsymbol{v}_{\text {कout }}}{v_{\infty}^{2}}\right) \\
& =\arccos \left(\frac{V \sin \beta \cos \psi-L \cos \beta}{v_{\infty}}\right)
\end{aligned}
$$

The angle $\varphi$ is defined as shown in Fig. 5 to correlate $\psi$ to B-plane parameter $\theta$. The angle $\varphi$ is measured from the $P_{1}$ axis to a mapped trace of $\boldsymbol{v}_{\boldsymbol{c} \text { out }}$ on the $P_{1} P_{3}$ plane. From Eq. (7), parameter $\varphi$ that corresponds to each $\psi$ is derived as

$$
\begin{aligned}
& \cos \varphi=\frac{V \sin \psi}{\sqrt{(V \sin \psi)^{2}+(L \sin \beta+V \cos \beta \cos \psi)^{2}}} \\
& \sin \varphi=\frac{L \sin \beta+V \cos \beta \cos \psi}{\sqrt{(V \sin \psi)^{2}+(L \sin \beta+V \cos \beta \cos \psi)^{2}}} .
\end{aligned}
$$




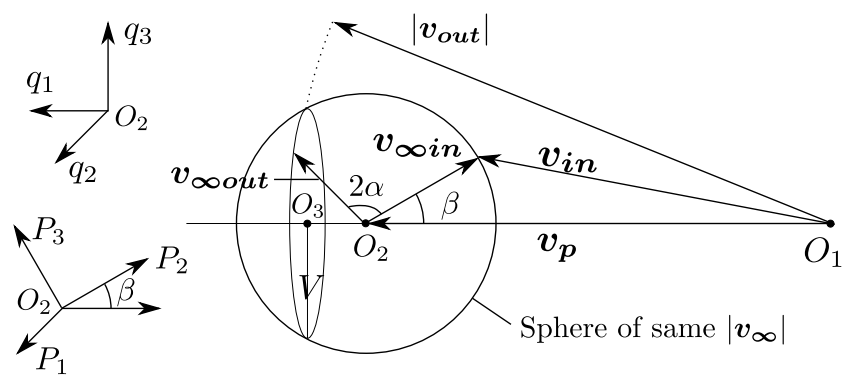

Fig. 2. Relation among velocity vectors at the swing-by.

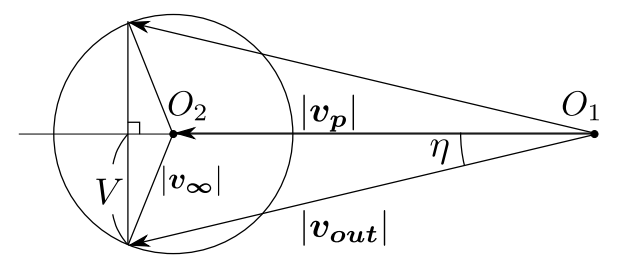

Fig. 3. Two-dimensional geometry of velocity vectors at the swing-by.

To associate the B-plane coordinate system with the $\mathrm{O}_{2}-$ $P_{1} P_{3}$ coordinate system, we introduce another parameter $\varphi_{R}$. As shown in the bottom right picture in Fig. 5, $\varphi_{R}$ is determined from the following equations

$$
\cos \varphi_{R}=\hat{\boldsymbol{R}} \cdot \hat{\boldsymbol{P}}_{\mathbf{1}} \quad, \quad \sin \varphi_{R}=\hat{\boldsymbol{R}} \cdot \hat{\boldsymbol{P}}_{\mathbf{3}},
$$

and then $\theta$ for each parameter $\psi$ is computed as

$$
\theta=\frac{3}{2} \pi+\varphi_{R}-\varphi
$$

The value of $b$ is calculated from Eqs. (8), (12), and (13). ${ }^{8)}$

$$
\begin{gathered}
\sin \alpha=\frac{1}{1+\frac{r_{\text {peri }} v_{\infty}^{2}}{\mu_{p}}} \quad\left(0^{\circ} \leq \alpha \leq 90^{\circ}\right) \\
b=r_{\text {peri }} \sqrt{1+\frac{2 \mu_{p}}{r_{\text {peri }} v_{\infty}^{2}}}
\end{gathered}
$$

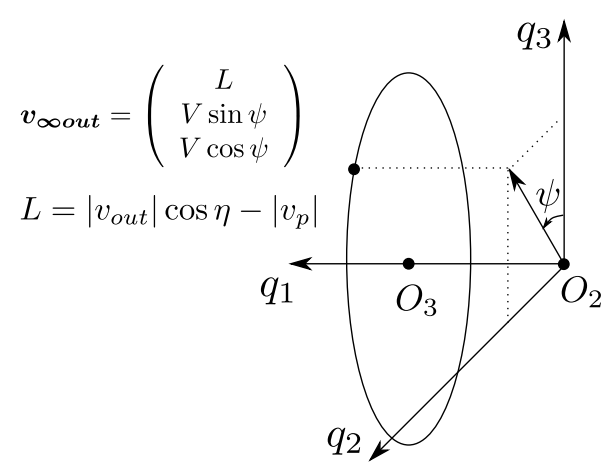

Fig. 4. $v_{\infty \text { out }}$ in the $O_{2}-q_{1} q_{2} q_{3}$ coordinate system.

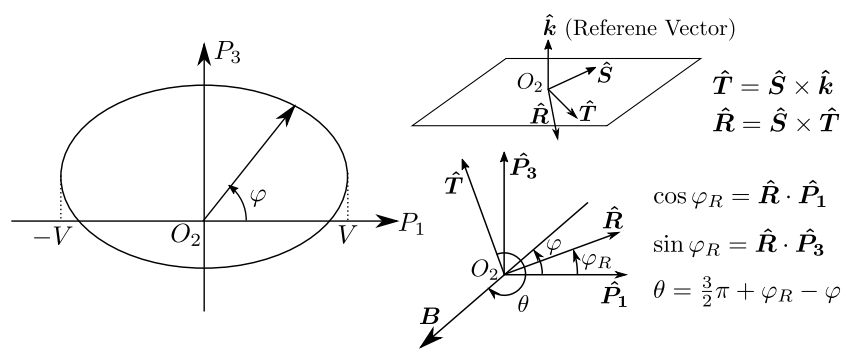

Fig. 5. Mapping of $v_{\infty \text { out }}$ onto the $P_{1} P_{3}$ plane.

\section{General Characteristics of FR Ensured Orbit Insertion}

\subsection{Reachability of the FR trajectory}

How acutely a body can bend a spacecraft's trajectory depends on $R_{p}$ and $\mu_{p}$. A smaller radius enables a close approach to the mass center, and a body with larger $\mu_{p}$ can bend the trajectory more easily, both of which lead to an increase in possible maximum deflection angle at the swing-by. Whether we can use the method of FR ensured orbit insertion depends on the deflection angle $\alpha_{F R}$ and $\alpha_{\max }$ at a given approaching velocity. In this section, we generally characterize the reachability of the FR trajectory after a swing-by. To make the system time-independent, the velocity of the planet is assumed to be constant; the velocity of each planet is computed as a velocity of a circular orbit with radius $a_{p}$. This assumption becomes valid when the eccentricity of the planet is close to zero.

\subsection{Scaling}

When we choose scaling factors for nondimensionalization as follows, $\alpha_{\max }$ and $\alpha_{F R}$ are characterized by a single parameter $\lambda$ associated with the target body.

$$
\begin{gathered}
L_{s f}=R_{p} \quad \text { (radius of the target body) } \\
T_{s f}=\sqrt{\frac{R_{p}^{3}}{\mu_{p}}}
\end{gathered}
$$

The upper bound is given as

$$
\alpha_{\text {max }}=\arcsin \left(\frac{1}{1+v_{\infty}^{* 2}}\right),
$$

and $\alpha_{F R}$ is obtained from the following equations.

$$
\begin{gathered}
2 \alpha_{F R}=\arccos \left(\frac{V^{*} \sin \beta \cos \psi-L^{*} \cos \beta}{v_{\infty}^{*}}\right) \\
\cos \eta=\frac{v_{\text {out }}^{* 2}+v_{p}^{* 2}-v_{\infty}^{* 2}}{2 v_{\text {out }}^{*} t_{p}^{*}} \\
\sin \eta=\sqrt{1-\cos \eta^{2}} \\
V^{*}=v_{\text {out }}^{*} \sin \eta \\
L^{*}=v_{\text {out }}^{*} \cos \eta-v_{p}^{*} \\
v_{\text {out }}^{*}=\sqrt{\lambda\left(2-\left(\frac{N}{M}\right)^{\frac{2}{3}}\right)} \\
v_{p}^{*}=\sqrt{\lambda} \\
\lambda=\frac{R_{p} \mu_{\text {Sun }}}{a_{p} \mu_{p}}
\end{gathered}
$$

The parameter $\lambda$ defined in Eq. (24) becomes the square of the ratio of the velocity of the planet to the first astronautical velocity around the planet. A small $\lambda$ corresponds to a large first astronautical velocity for the given $v_{p}$. If the first astronautical velocity is relatively large, a spacecraft can attain high speed at the periapsis for the same $v_{\infty}$, which results in the large $\alpha_{\max }$. Therefore, $\lambda$ signifies how hard it is to deflect the trajectory of a spacecraft. We can use FR ensured orbit insertion if the condition $\alpha_{F R} \leq \alpha_{\max }$ is satisfied. 
Table 1. Body name and corresponding parameter $\lambda$.

\begin{tabular}{cccc} 
Target & Primary & $\lambda$ & $\begin{array}{c}v_{\infty}^{*} \text { for Hohmann transfer } \\
\text { from Earth }\end{array}$ \\
\hline & & & $\begin{array}{c}3.89 \times 10^{-1} \\
\text { (from LEO) }\end{array}$ \\
Moon & Earth & $3.67 \times 10^{-1}$ & 3.20 \\
Mercury & Sun & $2.54 \times 10^{2}$ & $3.70 \times 10^{-1}$ \\
Venus & Sun & $2.28 \times 10$ & - \\
Earth & Sun & $1.42 \times 10$ & $7.46 \times 10^{-1}$ \\
Mars & Sun & $4.62 \times 10$ & $1.34 \times 10^{-1}$ \\
Jupiter & Sun & $9.62 \times 10^{-2}$ & $2.17 \times 10^{-1}$ \\
Saturn & Sun & $1.48 \times 10^{-1}$ & $3.09 \times 10^{-1}$ \\
Uranus & Sun & $2.04 \times 10^{-1}$ & $2.44 \times 10^{-1}$ \\
Neptune & Sun & $1.07 \times 10^{-1}$ & \\
\hline
\end{tabular}

\subsection{FR ensured polar orbit insertion}

When we plan an orbit insertion, its target trajectory is decided so that the trajectory meets mission requirements and, at the same time, has small $\Delta V$. It is conceivable that there is a trade-off between the condition for FR ensured orbit insertion and required $\Delta V$. With the intent to evaluate the trade-off in a general manner, an orbit insertion to polar orbit is discussed in this section.

The reason we choose a polar orbit as a test case is that its simple formulation is suitable for investigating general characteristics of FR ensured orbit insertion. The study on the polar orbit insertion is also useful from the viewpoint of actual mission application. In a planetary exploration mission, the polar orbit is often profitable, for a spacecraft observes the entire surface of the body as a result of its rotation. When a spacecraft approaches a target body, there are always regions where we can insert a spacecraft into the polar orbit, regardless of its approaching direction. For instance, if a reference vector that defines the B-plane is in a direction of the north pole, targeting any point on the $\hat{\boldsymbol{R}}$ axis results in the insertion to the polar orbit. Also, insertion $\Delta V$ depends only on the altitude where the spacecraft performs the maneuver; therefore, we can easily evaluate the $\Delta V$.

The FR ensured orbit insertion to the polar orbit is characterized by the geometric parameter $\varphi_{R}$ defined in Eq. (10). The parameter $\varphi_{R}$ indicates the direction of the south pole in the $P_{1} P_{3}$ plane when we choose $\hat{k}$ as a normal vector of the equatorial plane. By solving Eq. (7) for $\psi$, we can find $\psi_{R}$ corresponding to $\varphi_{R}\left(-\pi / 2<\varphi_{R}<\pi / 2\right)$ as follows.

$$
\begin{gathered}
\psi_{R}=\gamma+\arccos \left(\frac{L \sin \beta}{\sqrt{(V \cos \beta)^{2}+\left(V \tan \varphi_{R}\right)^{2}}}\right) \\
\text { where }\left\{\begin{array}{l}
\cos \gamma=\frac{-V \cos \beta}{\sqrt{(V \cos \beta)^{2}+\left(V \tan \varphi_{R}\right)^{2}}} \\
\sin \gamma=\frac{V \tan \varphi_{R}}{\sqrt{(V \cos \beta)^{2}+\left(V \tan \varphi_{R}\right)^{2}}}
\end{array}\right.
\end{gathered}
$$

The tracks of $\boldsymbol{v}_{\text {cout }}$ are symmetric against the $P_{3}$ axis as seen in Fig. 5. Therefore, examining the range of $-\pi / 2 \leq \varphi_{R} \leq \pi / 2$ suffices to know the entire solution space.

Once $\varphi_{R}$ is specified, $\psi_{R}$ is obtained. Then, $\alpha_{F R}$ is obtained by Eqs. (17) to (24). By providing two parameters $\varphi_{R}$ and $\lambda$, one can judge if $\alpha_{\max }$ is larger than $\alpha_{F R}$ for each incoming $\boldsymbol{v}_{\infty}$. Maps that represent possible FR trajectories are obtained by this method, using $v_{\infty}$ and $\beta$ as independent variables. To understand

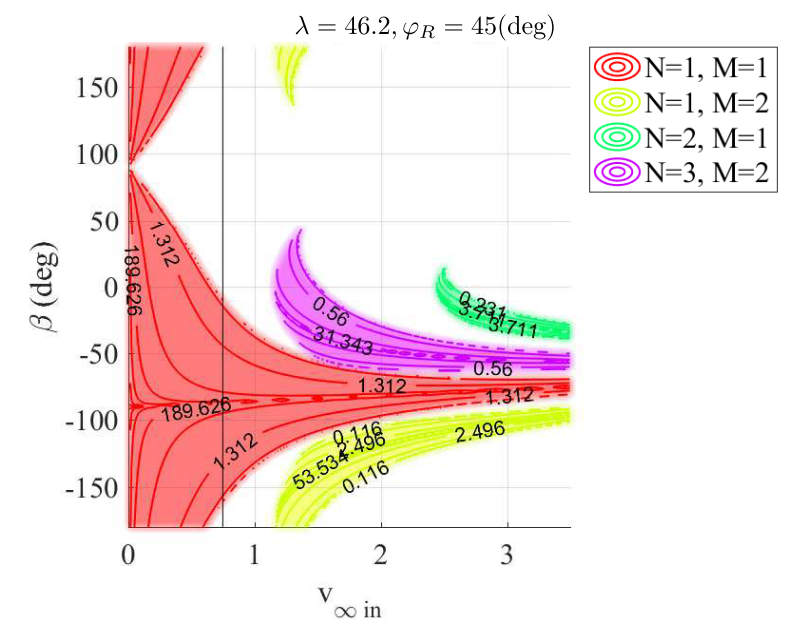

Fig. 6. Reachability of FR ensured orbit insertion over $v_{\infty}$ and angle $\beta$ for Mars. $\lambda=46.2$ and $\varphi_{R}=45 \mathrm{deg}$.

the dependency on the parameters $\varphi_{R}$ and $\lambda$, maps with $\lambda=1,5$, and 100 are shown for each $\varphi_{R}=0 \mathrm{deg}, 10 \mathrm{deg}$, and $45 \mathrm{deg}$ in Fig. 7. In the figure, only the combinations of $\{\mathrm{N}: \mathrm{M}\}=\{1: 1\},\{1: 2\}$, $\{2: 1\}$, and $\{3: 2\}$ are displayed. As $\lambda$ becomes small, overlaps of reachable regions for a different combination of $\{\mathrm{N}: \mathrm{M}\}$ emerge. The maps with negative and positive $\varphi_{R}$ are symmetric against a line of $\beta=0$. This symmetry is apparent when we take a look at Fig. 2. The value of $\alpha$ corresponding to a given $\varphi_{R}$ and $\beta$ is same for $-\varphi_{R}$ and $-\beta$. In Fig. 6 , we overlay contour lines on the reachable region that signify a non-dimensional altitude from a target body's surface at the periapsis. This altitude is computed from the non-dimensional version of Eq. (12). The information on the altitude is helpful in estimating necessary $\Delta V$ for the orbit insertion. As $\lambda$ gets smaller, the possible region for FR ensured polar orbit insertion forms a closed shape as in the second row of Fig. 7. The value of the contour is small in the area near the center of this closed shape.

In Fig. 6, the detailed map for Mars orbit insertion is shown. Values of $v_{\infty}$ for Hohmann transfer from Earth are expressed as a black line. The values of $\lambda$ and the characteristic $v_{\infty}$ for Hohmann transfer from Earth are summarized in table 1. In the case of polar orbit insertion, necessary $\Delta V$ depends only on the altitude from the target body, given $v_{\infty}$. By looking at the map, we can determine ideal $v_{\infty}$ and $\beta$ that satisfy the FR condition and small $\Delta V$. Even in the other type of the orbit insertion, low altitude at periapsis generally contributes to the small $\Delta V$ for the insertion. Thus, the map also serves as a reference to estimate a desirable approaching condition beyond the polar orbit insertion.

\section{Application to MMX Mission}

The Martian Moons eXplorer (MMX) mission is a sample return mission from one of the Martian Moons, Phobos. JAXA has planned to launch the probe in the early 2020s. In the design of a trajectory that is tolerant to an orbit insertion failure, a key question is whether we can achieve robustness and small $\Delta V$ at the same time. Since the explorer needs to visit Phobos, it executes multiple maneuvers to alter its trajectory. Necessary $\Delta V$ for the orbit insertion does not monotonously change according to the distance from Mars on the B-plane, 
Trans. JSASS Aerospace Tech. Japan Vol. 17, No. 3 (2019)
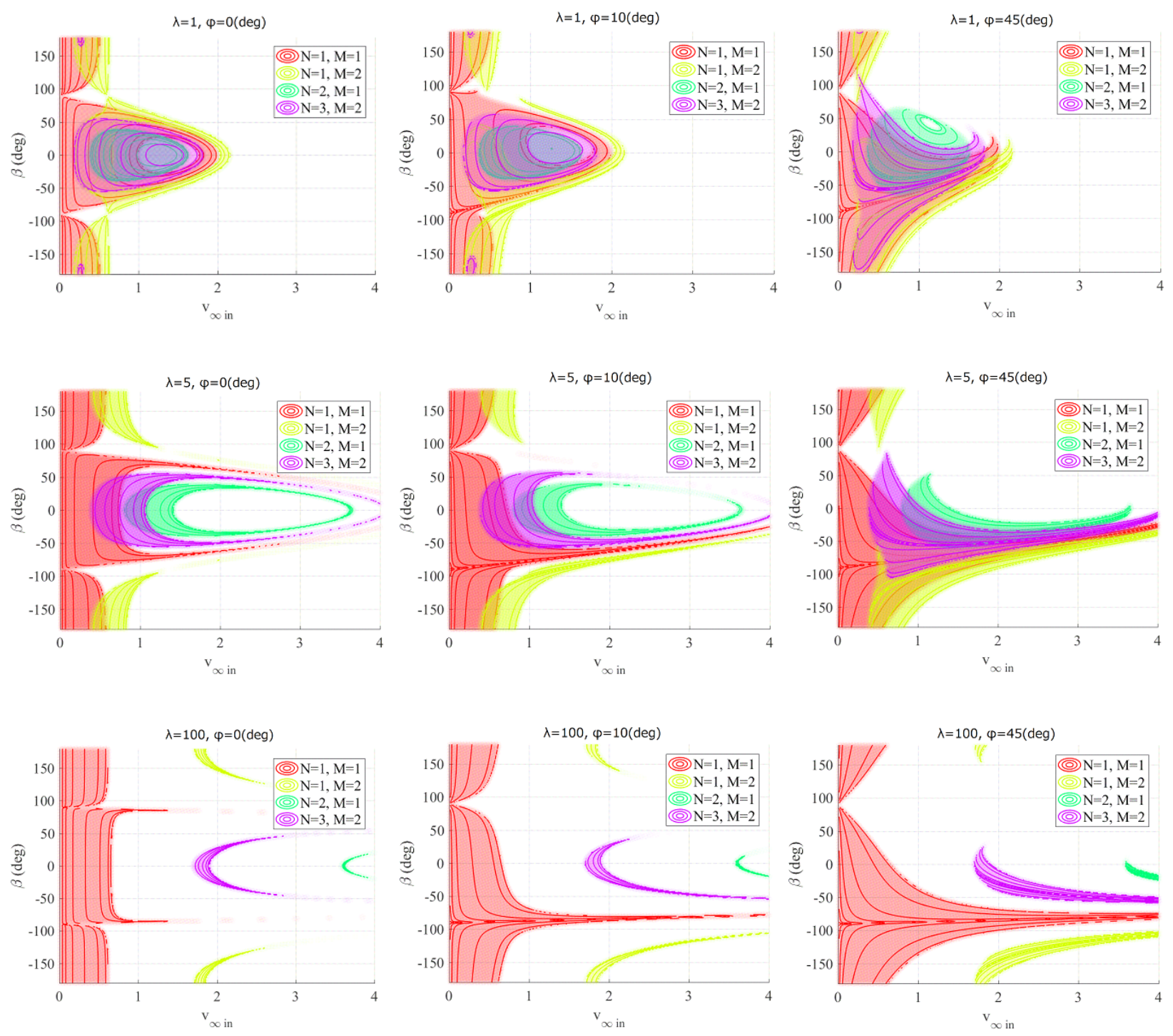

Fig. 7. Reachability of FR ensured orbit insertion over $v_{\infty}$ and angle $\beta$. Body associated parameter $\lambda$ increases from top to bottom $(1,5,100)$. Geometric parameter $\varphi_{R}$ increases from left to right $(0,10 \mathrm{deg}, 45 \mathrm{deg})$.

which is a major difference with the polar orbit insertion.

From Fig. 6, we will see that a line showing the $v_{\infty}$ for Hohmann transfer is in the region of 1:1 FR trajectory when $\varphi_{R}$ is large. Since MMX uses a trajectory like Hohmann transfer, we choose 1:1 FR trajectory for the target of the FR ensured orbit insertion. If the insertion maneuver fails, the probe can re-encounter Mars after one Martian year (1.88 Earth years).

An overview of the trajectory design is as follows. ${ }^{9)}$ In the preliminary design, the use of a chemical propulsion system and an electric propulsion system were investigated, and the plan to use the chemical propulsion system for the entire mission has been adopted. In the reported nominal condition, the spacecraft departs from Earth on September 11 in 2024 and arrives at Mars about a year later. After the spacecraft arrives at the periapsis of Mars, it will perform three maneuvers for the Mars orbit insertion (MOI) and will transfer to the orbit of Phobos. Ogawa et al. have explained the nominal sequence of MOI as follows.

- MOI1

At the periapsis, MOI1 decelerates the explorer with respect to Mars and injects it into the elliptic orbit around
Mars. The altitude at the periapsis is $500 \mathrm{~km}$ from the ground and radius at the apoapsis is $40 R_{M}$. This initial elliptic trajectory is designed so that its apoapsis is in the orbital plane of Phobos, which means the argument of periapsis $\omega$ regarding Phobos's orbital plane is either $180^{\circ}$ or $0^{\circ}$.

- MOI2

At the apoapsis where the orbital plane of Phobos and the orbital plane of the spacecraft intersect, MOI2 is performed. This maneuver changes its orbital plane to that of Phobos and lifts the altitude at the periapsis to the orbital radius of Phobos.

- $\mathrm{MOI} 3$

At the periapsis, MOI3 is applied and lowers the apoapsis altitude. Ogawa et al. have explained that the merit of choosing the specific argument of periapsis $\omega$ is that it reduces $\Delta V$ for MOI2 since the plane change can be achieved when the spacecraft has the lowest velocity. ${ }^{9)}$

For the computation of MOI, a similar sequence is adopted in this study. To enable the computation for any value of $b$ and 


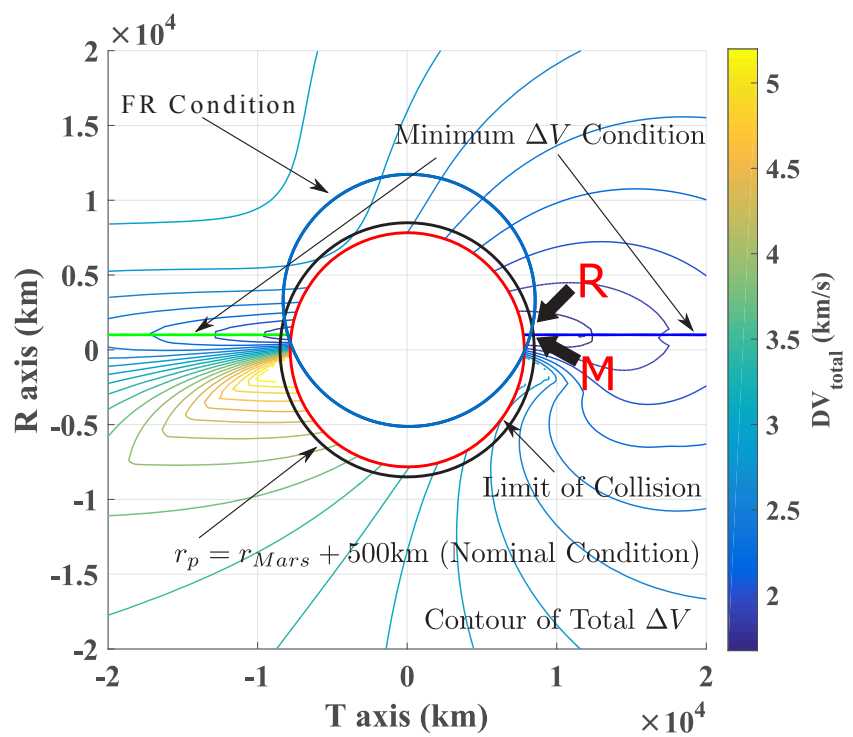

Fig. 8. Conditions on the B-plane for the nominal launch and arrival case.

$\theta$, we assume that MOI2 is performed at either an ascending or descending node. Except for MOI2, the scenario is the same.

In this study, the trajectory of the spacecraft is computed in the two-body model. The states of planets are retrieved from ephemerides.

\subsection{FR condition and $\Delta V$ on the B-plane}

Figure 8 shows the contour of necessary total $\Delta V$ at MOI on the B-plane for the nominal launch case; also depicted are the nominal altitude (black circle), the FR condition (blue circle), and the minimum $\Delta V$ condition (blue and green line). We define the minimum $\Delta V$ condition as the condition where $\omega$ after MOI1 is either $0^{\circ}$ or $180^{\circ}$. In this case, the spacecraft accomplishes the plane change at the lowest speed.

If we fix the altitude at the periapsis to $500 \mathrm{~km}$, the target point is on the black circle in the figure. An intersection of the blue line and the black circle (point M) is the potential solution if we do not take robustness into account. When we give priority to

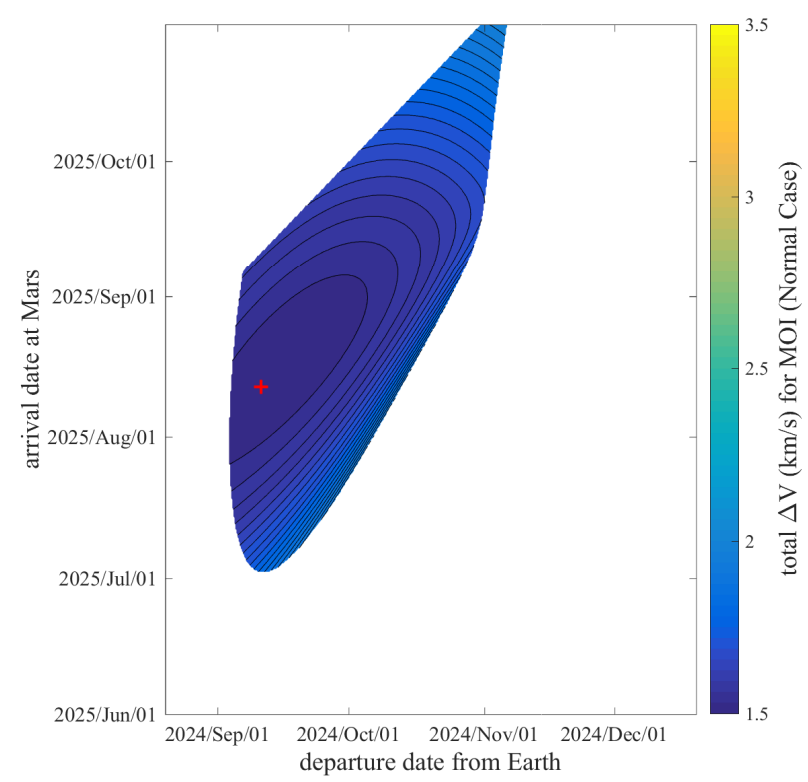

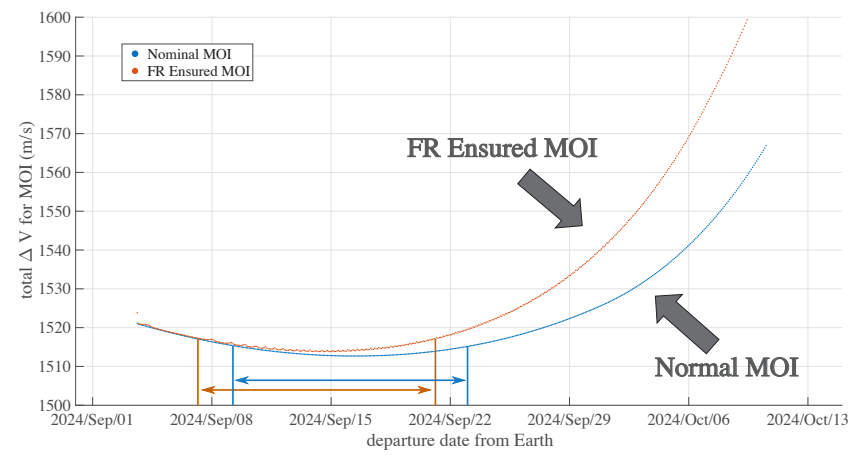

Fig. 10. Total $\Delta V$ at MOI for each launch date.

the FR ensured insertion, the target point is the intersection of the blue and black circle (point R). Usually, the point $\mathrm{M}$ and $\mathrm{R}$ do not coincide, which means the additional $\Delta V$ is required for FR ensured MOI. The amount of drawback changes depending on the launch and arrival date. Therefore, we investigated the necessary drawback for the FR ensured orbit insertion for each launch and arrival date.

\subsection{Determining the launch window}

Figure 9 shows the porkchop plot of total $\Delta V$ for MOI. The left and right figures correspond to normal the MOI and the FR ensured MOI respectively. A red plus mark is the current nominal. In the computation, the radius at MOI1 is fixed to $R_{M}+500 \mathrm{~km}$ both in the FR and non-FR ensured MOI. In the design of the nominal condition by Ogawa et al., mission requirements are defined as shown below. ${ }^{9)}$ We follow the same requirement for the trajectory design with FR ensured MOI. In the porkchop plot, launch and arrival conditions that do not satisfy those requirements are excluded.

- $C_{3}$ at Earth is smaller than $18 \mathrm{~km}^{2} / \mathrm{s}^{2}$.

- The declination of $\boldsymbol{v}_{\infty}$ at Earth satisfies $-30^{\circ} \leq \delta \leq 30^{\circ}$.

This condition is required for the lunch from Tanegashima Space Center.

- The flight time is less than one year.

By looking at the porkchop plot, we can see that the $\Delta V$ for FR

Fig. 9. Porkchop plot of total $\Delta V$ for MOI in case of normal MOI. The left and right figure correspond to normal MOI and FR ensured MOI respectively. 
ensured MOI can be as small as normal MOI if proper launch and arrival conditions are picked.

According to Ogawa et al., we must secure at least 2 weeks of consecutive launch window. ${ }^{9)}$ In this research, we design the launch window so that the worst value of $\Delta V$ at MOI becomes smallest in the two weeks. Figure 10 shows the $\Delta V$ at MOI for each launch date. The brown and blue plots represent FR ensured MOI and normal MOI respectively. The launch windows are also depicted for both normal and FR ensured MOI. From the figure, it can be seen that the difference of the worst case $\Delta V$ between the two launch windows is less than $10 \mathrm{~m} / \mathrm{s}$. Therefore, adopting FR ensured MOI has little impact on the $\Delta V$ budget.

\section{Discussions}

In this research, we focused only on the case where a spacecraft completely fails orbit insertion maneuver. However, a thrust which is performed but not sufficient is also probable. In this case, target point on the B-plane that achieves FR condition changes depending on how unsuccessful the maneuver is. One method to design FR ensured orbit insertion in this case would be to target a point on the B-plane that minimizes the expected value of the total $\Delta V$ from the initial insertion to final target orbit. We define $f$ as the success rate of the initial orbit insertion maneuver. The value of $f$ is between 0 and 1 each corresponding to the complete failure and nominal orbit insertion.

$$
f=\frac{\text { Initial } \Delta \mathrm{V} \text { (actually performed) }}{\text { Initial } \Delta \mathrm{V} \text { (nominal value) }}
$$

We also assume that a probability distribution is given as a density function $P(f)$. If we define a scenario where a spacecraft retries orbit insertion, initial target point can be computed as $b$ and $\theta$ from the following formulation.

$$
\min _{b, \theta} J(b, \theta)=\int_{0}^{1} P(f) \Delta V(b, \theta, f) d f
$$

Here, $\Delta V(b, \theta, f)$ is the total $\Delta V$ from the initial approaching trajectory to the final target orbit. Once $\Delta V(b, \theta, f)$ is defined, we can compute the optimal $b$ and $\theta$ for any form of probability distribution.

We tried applying this idea to the MMX mission. The detail about the computation and obtained preliminary results are placed in the appendix since the discussion is off the main topic.

The track of optimal point when we change probability distribution or spacecraft's approaching velocity is still to be investigated. Also, further investigation is necessary on how to decide the optimized scenario to re-target the orbit after an orbit insertion failure.

\section{Conclusions}

We investigated the general characteristics of FR ensured orbit insertion and its application to JAXA's MMX mission. Conclusions are as follows.

We established the analytical method to compute target points for FR ensured orbit insertion on the B-plane. The values of $b$ and $\theta$ that satisfy the FR condition are obtained based on the geometry of the velocity vectors at the swing-by. Using the coordinate transformation, $\boldsymbol{v}_{\boldsymbol{c} \text { out }}$ was expressed by a single parameter $\psi$. For each $\psi$, the value of $b$ is obtained through the computation of deflection angle $\alpha$ and the radius at periapsis $r_{\text {peri }}$. The angle $\theta$ that shows the direction of insertion is also computed by correlating the reference frame $O-P_{1} P_{3}$ and the B-plane.

By properly choosing the scaling factors as $R_{p}$ and $\sqrt{R_{p}^{3} / \mu_{p}}$, we showed that a maximum deflection angle achieved by the planet $\alpha_{\text {max }}$ and a necessary deflection angle for the FR ensured polar orbit insertion $\alpha_{\text {robsut }}$ are classified by two parameters $\lambda$ and $\varphi_{R}$. We showed that $\lambda$ works as an index to show how difficult it is to deflect a trajectory at the swing-by and $\varphi_{R}$ works as a parameter to correlate the insertion direction on the B-plane.

General maps that show the conditions on incoming $\boldsymbol{v}_{\infty}$ to achieve an FR ensured polar orbit insertion were obtained for each set of two parameters $\lambda$ and $\varphi_{R}$. We presented that by looking at the maps, desired approaching condition can be evaluated and mission planning would benefit from it.

The method of FR ensured orbit insertion is applied to the trajectory design of MMX mission and we confirmed that we can secure two weeks of the launch window that satisfies the mission requirements with the extra $\Delta V$ for MOI being as small as several $\mathrm{m} / \mathrm{s}$.

To expand FR ensured orbit insertion, consideration to the maneuver that stops in the middle is necessary. We will be able to adopt the approach based on the probability distribution of the success rate at initial maneuver. The relation between the optimal target point and the change of probability distribution is still to be investigated. Also, properly choosing the scenario after the insertion failure requires further work.

\section{References}

1) Holdridge, M.: NEAR Shoemaker Spacecraft Mission Operations, Johns Hopkins APL Technical Digest, 23 (2002), pp. 58-70.

2) Bell, J. and Mitton, J.: Asteroid Rendezvous: NEAR Shoemaker's Adventures at Eros. Cambridge University Press, Cambridge, 2002, pp. 15-18.

3) Hirose, C., Ishii, N., Yamamoto, T., Kawakatsu, Y., Ukai, C., Terada, H., and Ebara, M.: The Trajectory Design Strategies for Akatsuki Mission, Trans. JSASS Aerospace Technology Japan, 10, ists28 (2012), pp. To_3_1-To_3_5.

4) Kawakatsu, Y., Yamamoto, M., and Kawaguchi, J.: Study on a Lunar Approaching Strategy Tolerant of a Lunar Orbit Injection Failure, Trans. JSASS Aerospace Technology Japan, 5 (2007), pp. 1-7.

5) Russell, R. and Ocampo, C.: Geometric Analysis of Free-Return Trajectories Following a Gravity-Assisted Flyby, J. Spacecraft Rockets, 42 (2005), pp. 138-151.

6) Strange, N., Russell, R., and Buffingtonm B.: Mapping the V-Infinity Globe, Proceedings of AAS Astrodynamics Specialist Conference, Mackinac Island, Michigan, USA, 2007, 07-277.

7) Kawakatsu, Y.: $v_{\infty}$ Direction Diagram and its Application to Swingby Design, Proceedings of 21st International Symposium on Space Flight Dynamics, Toulouse, France, 2009, 3.

8) Battin, H.: An Introduction to the Mathematics and Methods of Astrodynamics, AIAA Education Series, New York, 1987, pp. 419-436.

9) Ogawa, N., Tsuda, Y., Takei, Y., Inoue, H., Takahashi, S., and Kawakatsu, Y.: Orbit Design for Martian Moons Explorer, 31st International Symposium on Space Technology and Science, Matsuyama, Japan, ISTS-2017-d-012, 2017. 


\section{Appendix}

This appendix explains the detail on the expanded FR ensured MOI discussed in Section 5. Using the MMX mission as an example, the expected value of total $\Delta V$ is computed, assuming certain scenario after the failure. The preliminary results for the nominal launch and arrival condition are shown in the Fig. 11. The top and middle figures show the contour of the expected value of $\Delta V_{\text {actual }}$ on the B-plane when $P(f)$ is delta functions shown in the left figures. The bottom figure corresponds to the uniformly distributed $P(f)$, and the plot can be seen as the mixture of the top and middle figures. In this computation, following scenario is used. If a spacecraft is captured by MOI1, then MOI2 is performed at the intersection of the target orbital plane and the orbital plane of the spacecraft like the usual MOI2. The apoapsis altitude after the MOI1 is different from the nominal value. Then, MOI3 is applied at periapsis to lower the apoapsis altitude to the orbital radius of Phobos. If a spacecraft escapes from Mars, a deep space maneuver to re-target Mars is applied after 1 month later and the similar MOI maneuvers are performed at the second encounter.

This is just an example to demonstrate an approach to the problem and the method itself requires further discussions. For one thing, defining an optimized contingency scenario is not an easy task. For example, in the optimized recovery scenario, customizing the number and the timing of maneuvers will be necessary for each failure mode. For another, we must investigate the dependence of the optimal $b, \theta$, the expected value of $\Delta V$ at that point, or the sensitivity of $\Delta V$ on $P(f), \boldsymbol{v}_{\infty}$, or $\boldsymbol{v}_{p}$.
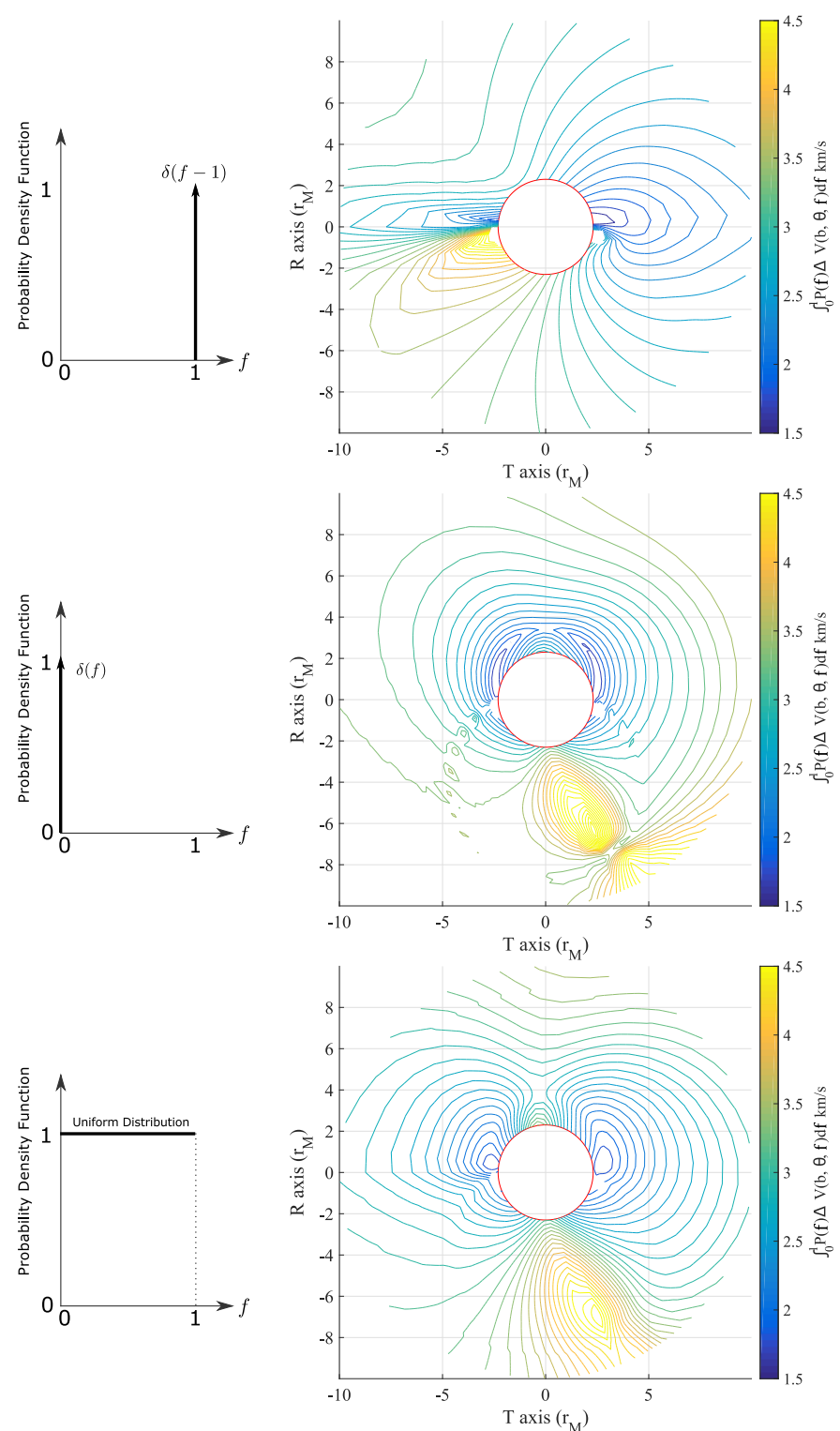

Fig. 11. Probability distribution and corresponding expected value of $\Delta V_{\text {actual }}$ on the B-plane. 